\title{
Is "Parkinson's disease" one disease?
}

\author{
DONALD B CALNE \\ From the Belzberg Laboratory of Clinical Neuroscience, Department of Medicine, Division of Neurology, \\ University Hospital, Vancouver, BC, Canada
}

SUMMARY Consideration is given to how and why categories of ill health are divided into diseases. Aetiology is a fundamental criterion for the delineation of individual diseases. The same clinical and pathological picture may have many different causes; for example meningococcal meningitis and pneumococcal meningitis are distinct diseases that may display the same symptoms and signs. On the other hand, a single aetiology may lead to quite separate clinical and pathological phenomena; for example, neurosyphilis is a disorder that can present with general paresis or tabes dorsalis (or any combination of the two). In attempting to find a nosological placement for Parkinson's disease, we must take into account the extensive overlap with idiopathic dementia (Alzheimer's disease). Present evidence raises the possibility of several causes for Parkinson's disease, some of which may also be responsible for idiopathic dementia. A classification in accord with its position is desirable, and as a first step it would be helpful to replace "Parkinson's disease" with a term that is not saddled with implications of a single causal mechanism. "Idiopathic Parkinsonism" is suitable nomenclature for what is really a syndrome of unknown origin.

This essay is an attempt to address the question "Is Parkinson's disease one disease?" From a practical viewpoint, a more important issue might seem "Does Parkinson's disease have one cause?", but these two questions are so closely linked that an approach to the former has profound implications for the latter. Indeed, the two questions are almost alternative formulations of the same problem. The central role of aetiology in the nosology of illness is illustrated by Joseph disease, where three clinically distinct syndromes are all designated as subtypes because genetic evidence shows that they have a shared origin. The situation becomes more complex when we consider idiopathic syndromes. To approach this issue a reasonable first step is a general consideration of how and why morbid entities are grouped, or separated. What factors underlie the classification of disease?

\section{The problem of classification}

Medical education focuses substantial attention on teaching the skills of identifying diseases and treating them. Much information is also provided on path-

Address for reprint requests: Dr D B Calne, Department of Medicine, Division of Neurology, University Hospital, 2211 Wesbrook Mall, Vancouver BC, V6T IW5, Canada.

Accepted January 1989 ology, epidemiology, and the natural history of diseases. Sparse consideration, if any, is given to the classification of diseases. Yet the whole framework of knowledge of modern medicine is based upon the scientific and philosophical tenets that ill health derives from a series of definable disturbances in the structure or function of the body. It is inferred that the various disorders can be grouped together according to similarities in clinical picture, similarities in mechanisms of causation, or similarities in anatomical, physiological, biochemical or pathological manifestations. It is helpful to illustrate this aspect of the nosology of diseases. For similarities of clinical picture, there are "the dementias," for similarities of causation "the hereditary ataxias", for similarities of anatomical disturbance "the extrapyramidal diseases," for similarities of physiological dysfunction "the epilepsies", for similarities of biochemical abnormality "the lipid storage diseases" and for similarities in pathology "the demyelinating diseases". Many other types of association between diseases exist, as in the "vascular diseases" the "dysimmune diseases," or the "infectious diseases". A group of diseases can also be defined by criteria from several different sources, for example the "axonal neuropathies" have clinical, physiological and pathological features in common. In contrast, the term "Parkinsonism" is employed for a constellation 
of disorders for which the only common denominator is the clinical picture.

From this inconsistent background of nosology, the relationships that hold the various clusters of diseases together can become difficult to discern. Diseases have been grouped wherever there are any common features that facilitate discussing them for the purposes of teaching, diagnosis, treatment, or research. But the factors that provide cohesion for each of these disciplines are totally different, so it is not surprising that the classification is so heterogeneous. The justification for the current situation is that the present nosology does "work" in the pragmatic sense that the groupings serve each purpose that has led to their formulation.

It is easier to deal conceptually with disorders if they are "lumped" rather than "split". For treatment, it is useful to have groups of illnesses that respond to related drugs such as steroids, antibiotics, or vitamins. For research it seems helpful to gather disorders together into categories that are accessible to similar methods of analysis.

By grouping morbid entities together we are making assumptions that may be quite unjustified. We cannot argue that all subjects with excessive neurofibrillary tangles and plaques in the brain have the same disease any more than we can conclude that all patients with excessive white cells in the cerebrospinal fluid have the same disease. However, we should not ignore the plaques and tangles; we need to know what causes them. We cannot assume that there is only one cause, yet that is the way we are conditioned to think if we group together those patients with dementia and excessive plaques and tangles under the heading of "Alzheimer's disease". We could be looking at a nonspecific pathological marker analogous to inflammatory cells. Equally, of course, we should not exclude the possibility that there is only one cause for the plaques and tangles; some markers, such as antibodies to motor end-plates, are highly specific.

The overlap between Parkinson's disease and Alzheimer's disease

The discussion can now be focused more sharply on Parkinsonism. We know that in many patients with advanced idiopathic Parkinsonism there is dementia with increased numbers of plaques and tangles in the brain at necropsy. From this finding should we argue: (1) That Parkinson's disease is a form of Alzheimer's disease? (2) that Alzheimer's disease is a form of Parkinson's disease? (3) that there is an association between Parkinson's disease and Alzheimer's disease? (4) that the usual criteria employed for defining these disease are unsatisfactory? (5) that the co-existence of Parkinson's disease and Alzheimer's disease occurs by chance? The problem is, of course, compounded by the fact that Lewy bodies, often regarded as the hallmark of Parkinson's disease, are also common in Alzheimer's disease.'

If we look for analogies to these problems, they are easy to find. Within neurology there are numerous circumstances where (1) different diseases present with similar syndromes and (2) different syndromes are caused by the same disease. For example:

\section{(1) Different diseases presenting with similar syn- dromes.}

(a) The causes of acute meningitis may be clinically indistinguishable. To discriminate between $H$. influen$z a e, N$ meningoccus and Str pneumoniae it is generally necessary to identify the organism in the cerebrospinal fluid. (b) A stroke may derive from a haemorrhage, thrombosis or embolism. Clinical separation may not be possible, so the neurologist frequently resorts to investigations such as magnetic resonance imaging.

(2) The same disease presenting with dissimilar syndromes

(a) Vitamin B12 deficiency may cause dementia, optic atrophy, subacute combined degeneration of the spinal cord, or a peripheral neuropathy. (b) Neurosyphilis can present with a meningovascular syndrome, tabes dorsalis or general paresis. This last example is particularly apposite. While the syndromes of tabes dorsalis and general paresis are quite distinct, and have their own characteristic neuropathology, they also occur together with sufficient frequency for the term "taboparesis" to have been coined. The analogy to Parkinson's disease and Alzheimer's disease is obvious.

To elucidate the nature of the problem further, we must examine the bricks that make up the edifice of our current classification of diseases, and pose the question "What constitutes a disease?"

\section{What constitutes a "disease?"}

Dictionaries define disease in terms of loss of health, and clearly infer that there must be symptoms. Dictionaries do not help us with the task of separating different diseases. However, a review of the medical literature over the last century does allow the formulation of a reasonably precise concept of the criteria that are likely to be most useful in defining a disease.

If we take examples of diseases which are reasonably well understood, such as Wernicke's encephalopathy or Wilson's disease, we can see how they emerge as distinct morbid entities. In these cases we can define a cause, a pathological picture, a pattern of clinical features, an age and sex distribution, a natural history, and a form of treatment. Standard texts will describe diseases under headings similar to this list of criteria.

Many disorders that are widely referred to as 
diseases do not possess these definable criteria, and in such cases it would seem more appropriate to employ the term "syndrome" rather than "disease". Examples include "Leigh's disease" and "Charcot-Marie-Tooth disease". Curiously, there seems to be no consistency in the history of nomenclature; for some disorders we have adopted an entirely precise and correct terminology, such as the Riley-Day syndrome or Ekbom's syndrome.

\section{One disease or several?}

It is remarkably difficult to find a clear statement of what constitutes Parkinson's disease. James Parkinson described a collection of associated clinical features ${ }^{2}$ and his nineteenth century colleagues named this constellation of symptoms and signs his "disease". We must modify the clinical picture painted by James Parkinson, to take account of experience occurring over a subsequent century. When we do this, it becomes evident that the clinical findings described by Parkinson occur in a settng where several different causes can be identified, so the clinical entity has been termed Parkinsonism or the Parkinsonian syndrome. Aetiologies for the syndrome include viral infection, hereditary disorders, and exposure to certain drugs or toxins. There remains a large group of patients for whom no such causal mechanism can be identified, and these have become the focus of our interest. ${ }^{3}$ If we accept the general criteria just proposed for defining a disease we can weigh the evidence for and against idiopathic Parkinsonism being one disease.

In support of one disease:

(1) There is a characteristic pattern of neuronal loss from the zona compacta of the substantia nigra. ${ }^{4}$

(2) The consequent depletion of striatal dopamine has a particular distribution such that putamenal involvement exceeds caudate loss. ${ }^{5}$

(3) Lewy bodies are found in the substantia nigra. ${ }^{6}$

(4) Symptoms are alleviated by increasing brain dopamine or administering dopamine substitutes. ${ }^{\text {? }}$

Against one disease:

(1) Cases of idiopathic Parkinsonism have been reported with neurofibrillary tangles but no Lewy bodies. ${ }^{8}$

(2) Lewy bodies are not specifically associated with idiopathic Parkinsonism; they are found in several neurological disorders, and even in normal elderly subjects. ${ }^{1}$

(3) The natural history of idiopathic Parkinsonism is highly variable, such that a benign form has been postulated to be distinguishable from a malignant form.'

(4) The extent of the therapeutic response to dopaminomimetics varies between patients with Parkinsonism, and is not specific; subjects with Parkinsonism induced by MPTP respond as well to levodopa as patients of comparable age with idiopathic Parkinsonism. ${ }^{10}$

(5) The distribution of striatal dopamine depletion (and by inference the distribution of nigral dopaminergic cell loss) may not be exclusive for idiopathic Parkinsonism, as a similar pattern has been seen in the single, so far analysed, case of Parkinsonism caused by manganese (Hornykiewicz, personal communication).

From a consideration of this analysis a picture begins to emerge in which the evidence assembled for each argument reflects a quantitative rather than a qualitative difference. Idiopathic Parkinsonism tends to have more consistent morphological and biochemical pathology, and there is a higher probability of a therapeutic response to dopaminomimetics. Are these distinctions sufficient to justify a firm separation of idiopathic Parkinsonism into one disease category? The evidence seems too insecure for such a definite conclusion. While the case is unproven, the position that we take probably depends on where our interests lie. For teachers, or practising clinicians, there is most to be gained by simplifying the issues and accepting the notion that idiopathic Parkinsonism is one disease. However, for those conducting research to elucidate the cause of idiopathic Parkinsonism, I would advocate a more cautious approach. We should acknowlege the complexity of the evidence and maintain an open mind. At present, there is insufficient evidence to allow the conclusion that idiopathic Parkinsonism is one disease.

On the one hand, there is adequate evidence to justify formulating an hypothesis that idiopathic Parkinsonism has multiple causes just as meningitis has more than one aetiology. On the other hand, we cannot exclude a concept of idiopathic Parkinsonism being one clinical facet of a neurodegenerative disorder than can also be expressed as idiopathic dementia (Alzheimer's disease), in a way analogous to the varied manifestations of neurosyphilis. These alternatives are not mutually exclusive. There may be several causes of idiopathic Parkinsonism, of which one (or more) is also responsible for idiopathic dementia. In my view, this last proposal seems to fit best the wide range of observations that have been claimed to shed light on the nature and cause of Parkinson's disease.

\section{References}

1 Gibb WRG. The epidemiology of Lewy bodies. In: Fahn S, Marsden CD, Calne D, Goldstein M, eds. Recent Developments in Parkinson's Disease. Volume II. Florham Park, New Jersey: McMillan Healthcare Information, 1987:1-13.

2 Parkinson J. An Essay on The Shaking Palsy. London: 
Whittingham and Rowland, 1817.

3 Duvoisin R. Genetics of Parkinson's disease. In: Streifler M, ed. Proceedings of the 9 th International Symposium on Parkinson's disease. New York: Raven Press, 1989. (In press.)

4 Hassler R. Zur Pathologie der Paralyse agitans und des postenzephalitischen parkinsonism. J Psychol Neurol (Lpz) 1938;48:387-476.

$5 \mathrm{Kish}$ SJ, Shannak K, Hornykiewicz O. Uneven pattern of dopamine loss in the striatum of patients with idiopathic Parkinson's disease: pathophysiologic and clinical implications. $N$ Engl J Med 1988;318:876-80.

6 Jellinger K. Pathology of Parkinson's syndrome. In: Calne DB, ed. Drugs for the treatment of Parkinson's disease. Handbook of Experimental Pharmacology. Vol
88. Berlin: Springer Verlag, 1989:47-112.

7 Calne DB. Drugs for the treatment of Parkinson's disease. Handbook of Experimental Pharmacology. Vol 88. Berlin: Springer Verlag, 1989:1-583.

8 Sukhaker S, Rajput AH, Rozdilsky B, Uitti RJ. A clinicopathologic study of neurofibrillary tangles in parkinsonism. Neurology 1987;37:278-9.

9 Birkmayer W, Reiderer P, Youdim MBH. Distinction between benign and malignant type of Parkinson's disease. Clin Neurol Neurosurg 1979;81:158-64.

10 Langston JW, Ballard P. Parkinsonism induced by 1methyl-4-phenyl-1, 2, 3, 6-tetrahydropropyridine (MPTP): Implications for the treatment and pathogenesis of Parkinson's disease. Can J Neurol Sci 1984;11:160-5. 